\title{
Long-term effect of the insoluble thread-lifting technique
}

\section{Mototsugu Fukaya}

Tsurumai Kouen Clinic, Nagoya, Japan
This article was published in the following Dove Press journal:

Clinical, Cosmetic and Investigational Dermatology

14 November 2017

Number of times this article has been viewed

Background: Although the thread-lifting technique for sagging faces has become more common and popular, medical literature evaluating its effects is scarce. Studies on its long-term prognosis are particularly uncommon.

Patients and methods: One hundred individuals who had previously undergone insoluble thread-lifting were retrospectively investigated. Photos in frontal and oblique views from the first and last visits were evaluated by six female individuals by guessing the patients' ages. The mean guessed age was defined as the apparent age, and the difference between the real and apparent ages was defined as the youth value. The difference between the youth values before and after the thread-lift was defined as the rejuvenation effect and analyzed in relation to the time since the operation, the number of threads used and the number of thread-lift operations performed.

Results: The rejuvenation effect decreased over the first year after the operation, but showed an increasing trend thereafter. The rejuvenation effect increased with the number of threads used and the number of thread-lift operations performed.

Conclusion: The insoluble thread-lifting technique appears to be associated with both early and late effects. The rejuvenation effect appeared to decrease during the first year, but increased thereafter. A multicenter trial is necessary to confirm these findings.

Keywords: insoluble thread, Aptos, thread-lift, Xtosis, Aptos spring

\section{Introduction}

The idea of lifting sagged faces noninvasively using medical threads is not novel. Serdev has been using elastic threads made of polycaproamide since 1994, and Sasaki reported using Gore-Tex cable sutures in 2003. ${ }^{1,2}$ Sulamanidze devised a barbed thread for tightening the facial tissue in 1998 and his product, called Aptos, has since become popular worldwide. ${ }^{3}$ Sulamanidze also invented a spring-formed thread made of polypropylene, termed Aptos Springs, in 2003. ${ }^{4}$ Various analogs of Aptos or Aptos Springs threads have been put forward and are currently available. ${ }^{5}$

The author has also performed minimally invasive face lifts on patients with sagged faces using a kind of Aptos analog, "Xtosis", which, like Aptos, is made of polypropylene (suture size 0 in USP) with barbs. The name Xtosis originated from its design similar to the letter X. Two threads are crossed at the temple site and the four ends of the two threads are pulled to tighten the tissue. The suture size of Xtosis is larger than that of the original Aptos thread to avoid breakage when it is pulled and tightened firmly. The author has also used one that is nearly identical to Sulamanidze's Aptos and Aptos
Correspondence: Mototsugu Fukaya Tsurumai Kouen Clinic, 5-20-6, Chiyoda, Nakaku, Nagoya 4600012 , Japan

$\mathrm{Tel}+8 \mathrm{I} 522640212$

Email moto@earth.ocn.ne.jp 
Springs analog, which is made of nylon and has a stronger bounce than polypropylene when shaped into a spring. ${ }^{12}$

Threads used for lifting can be made of insoluble and soluble material. The main advantage of using insoluble threads is their long-lasting effect, although clinical studies on this are scarce. The advantage of using soluble threads made of caprolactone or polydioxanone is that the complications are expected less frequently, especially from the viewpoint of long-term prognosis. ${ }^{6,7}$ This retrospective study investigated the long-term effects and complications of the thread-lifting technique using insoluble threads in patients with sagging facial tissue.

\section{Patients and methods}

Of the revisit patients seen from December 2016 to February 2017, 100 consecutive individuals who had previously undergone thread-lifting surgery were enrolled. Patients who had undergone a thread-lifting procedure within the past 2 months were excluded because there was a considerable return in the lifting effect immediately after the operation, which should not be considered as a true effect. Patients who underwent face-lift surgery or liposuction of the face or neck after thread-lift were also excluded. Patients who had a hyaluronic acid injection into the nasolabial fold over the last 12 months and lipolaser treatment of the neck or perioral region were enrolled, but analyzed separately afterward.
Photos of the patients were taken in the frontal and oblique views and cropped such that only the mid and lower face under the eyes was visible and used for evaluation. The area around the eyes was cropped off from the photos because signs of blepharoplasty or other treatments, including Botox injections, may influence the patient's age evaluation.

For recruiting women who would perform the assessment, 10 women (staff of the clinic) in their 40s-60s were tested for their ability to guess the patients' actual ages by viewing photos of individuals of various ages who have had no cosmetic treatment. Six of the 10 women whose assessment was closest to the actual age were selected as evaluators in the study. The photos of the 100 enrolled patients at the first and last visits (i.e., 200 photo sets in the frontal and oblique views) were randomly presented to the women who guessed the patients' ages. Each woman made one guess per patient. The six guessed ages were averaged, and the result was defined as the "apparent age" of the patients in the photo set. The difference between the patients' real age and apparent age was defined as the "youth value", and the difference between two youth values was defined as the "rejuvenation effect". For example, a 30-year-old patient, who had an apparent age of 31 years at her first visit, underwent a thread-lift. She returned, at her real age of 34 years, and was assigned an apparent age of 28 years. Her youth value changed from $30-31=-1$ to $34-28=6$. The rejuvenation effect was $6-(-1)=7$ (Figure 1).
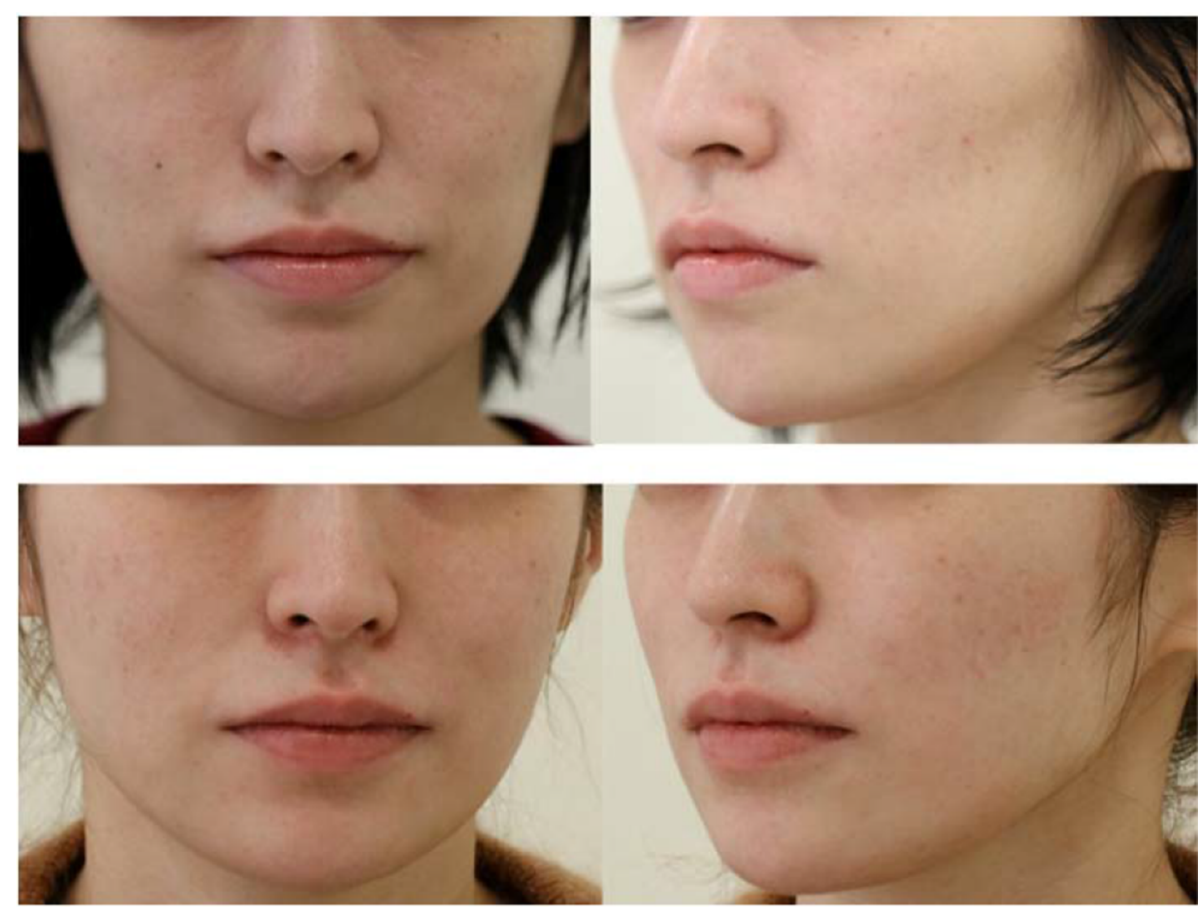

Figure I A photo example of an enrolled patient.

Notes: The upper photos are from the first visit at the age of 30, and the lower photos are of the last visit at the age of 34 . The patient underwent six thread-lifting surgeries and the total of 40 threads were inserted. The time from the last operation to the last visit was 9 months. Her apparent age was 31 at the first visit and 29 at the last visit. 
Written informed consent was obtained from all patients for this research, and also for the use of their images for evaluation and for the publication of the images.

\section{Results}

Of the 100 patients enrolled, the mean real ages at the first and last visits were $44.7 \pm 9.8$ (SD) and $49.6 \pm 10.8$ years, respectively. The mean apparent ages at the first and last visits were $45.0 \pm 10.9$ and $44.4 \pm 11.3$ years, respectively. The mean youth values at the first and last visits were $-0.3 \pm 5.6$ and $5.2 \pm 5.9$, respectively. There was a statistical significance $(P<0.0001$, paired $t$-test $)$ between the two youth values. Scatter plots of the patients' real and apparent ages at the first and last visits are shown in Figures 2 and 3. The youth value did not significantly differ between young and old patients.

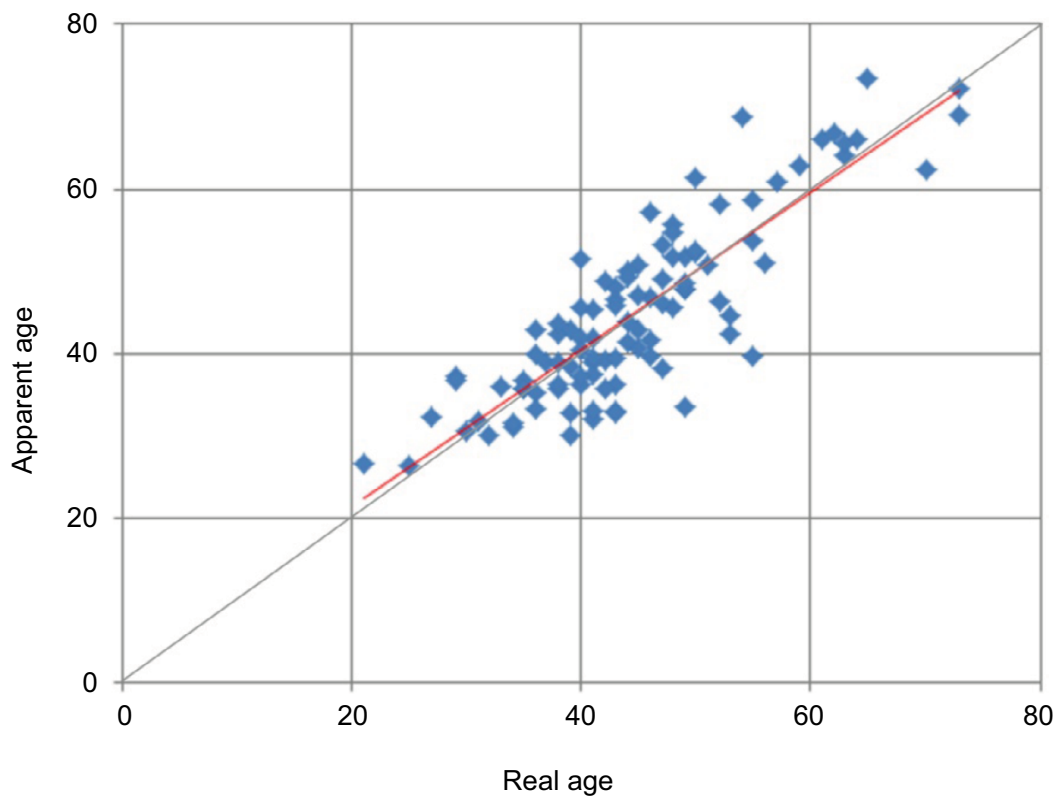

Figure 2 A scatter plot of the real ages and apparent ages of 100 patients at the first visit.

Note: The regression line is in red.

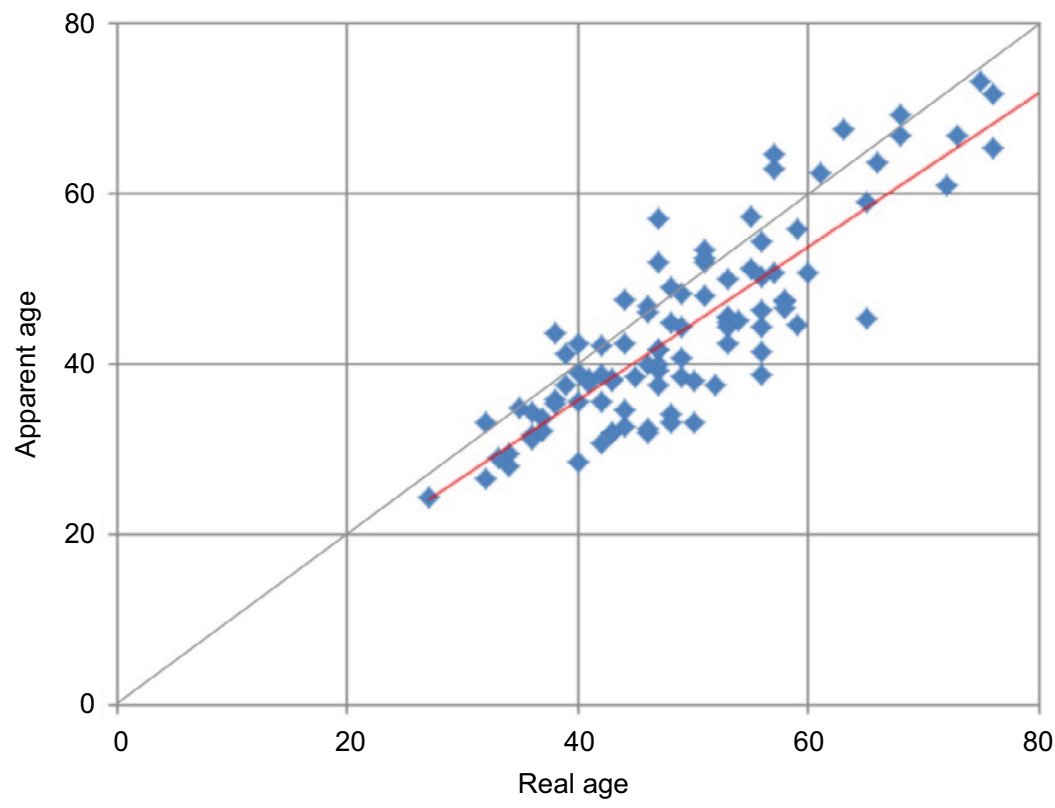

Figure 3 A scatter plot of the real ages and apparent ages of 100 patients at the last visit. Note: The regression line is in red. 
As for the time since the last thread-lift operation, 50 patients were seen $<1$ year after the procedure and the other 50 patients were seen $>1$ year after the procedure. The rejuvenation effects of these patients are plotted in Figures 4 and 5 , respectively. The regression line shows a decreasing trend in rejuvenation effects for the patients seen at $<1$ year after the last thread-lift procedure, whereas an increasing trend was noted for those seen $>1$ year after the last thread-lift procedure.
The mean number of procedures performed per patient was $2.9 \pm 3.1$, and the total number of threads inserted was 16.9 \pm 14 .4. The regression lines in Figures 6 and 7 show an increasing trend in both scatter plots.

Twenty-four out of 100 patients had hyaluronic acid injections to the nasolabial fold within 1 year before the last visit. These patients had a mean rejuvenation effect of $6.4 \pm 4.0$, while the rest of the study patients had $5.2 \pm 3.9(n=76)$. There was a weak statistical significance in the difference between

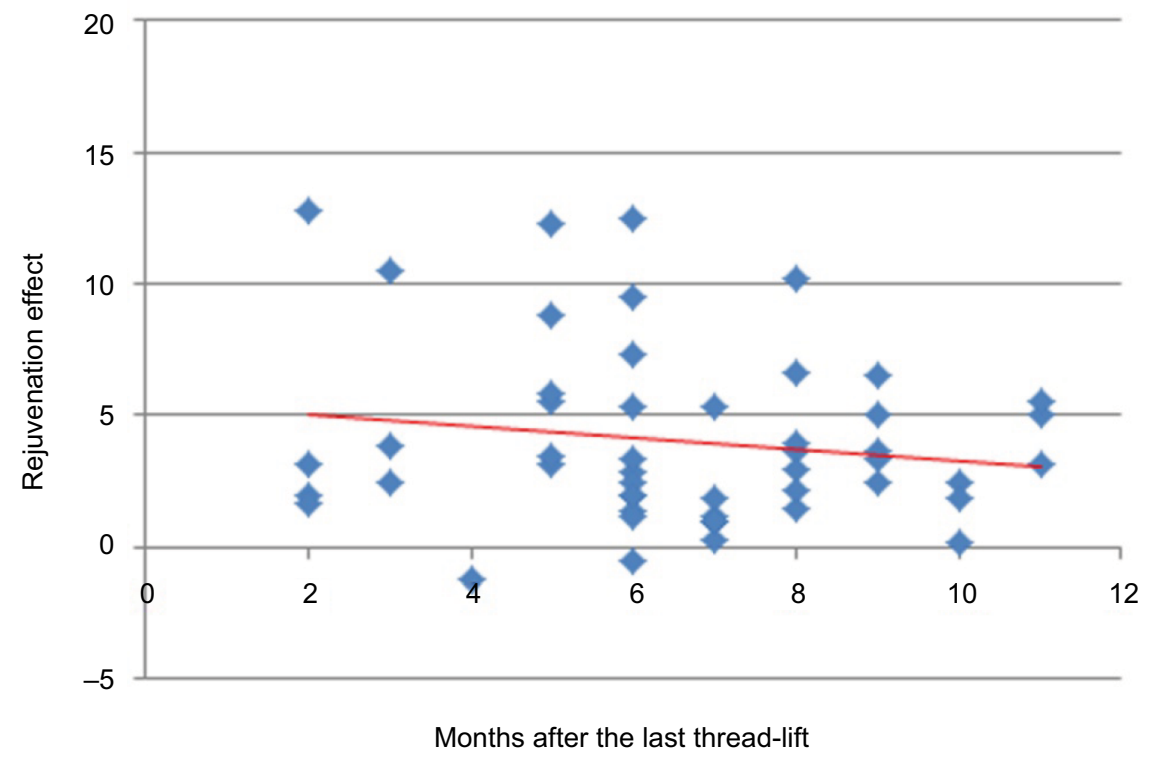

Figure $4 \mathrm{~A}$ scatter plot of the number of months since the last thread-lift and the rejuvenation effect in 50 patients. Note: The regression line is in red.

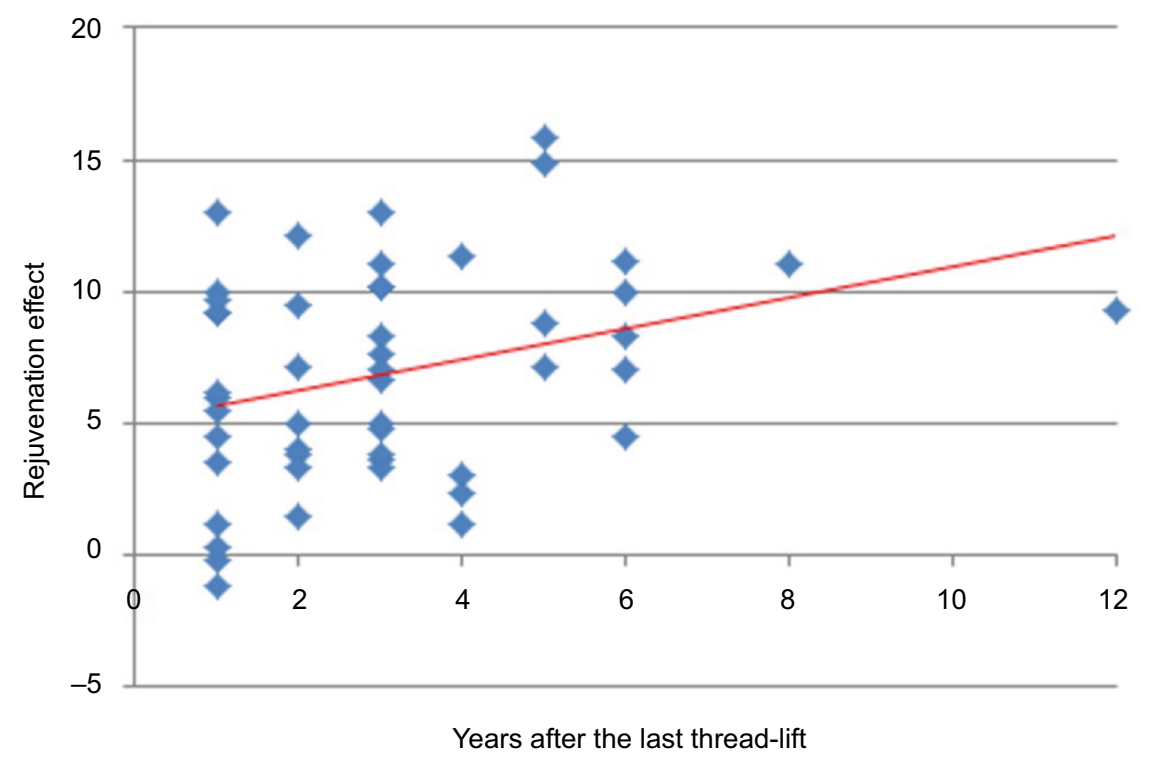

Figure $5 \mathrm{~A}$ scatter plot of the number of years since the last thread-lift operation and the rejuvenation effect in 50 patients. Note: The regression line is in red. 


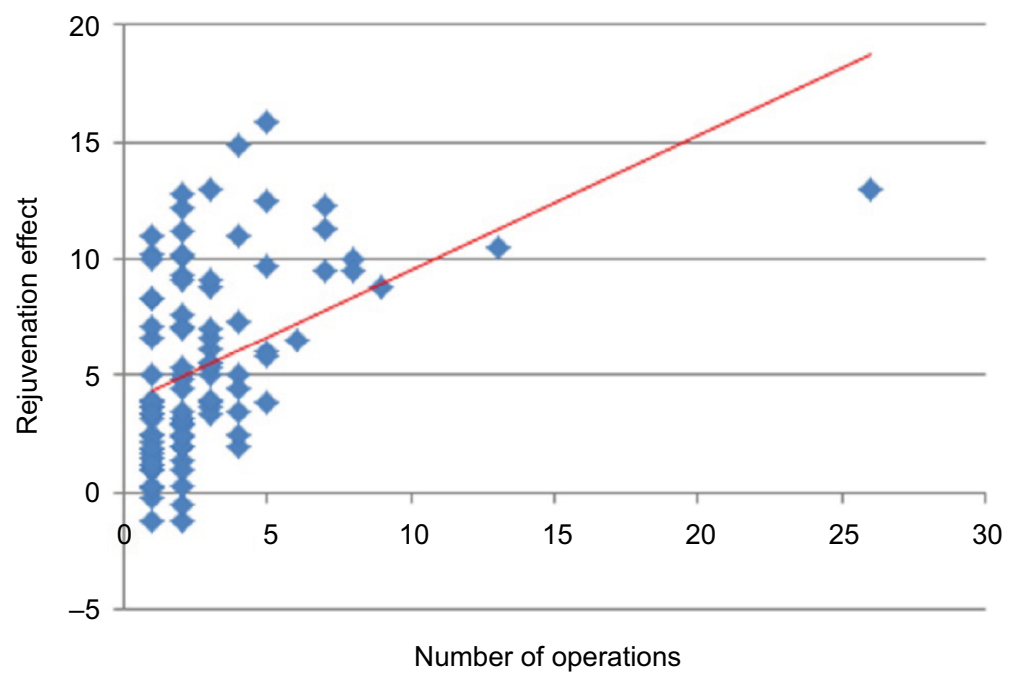

Figure 6 A scatter plot of the number of operations performed and the rejuvenation effect in 100 patients. Note: The regression line is in red.

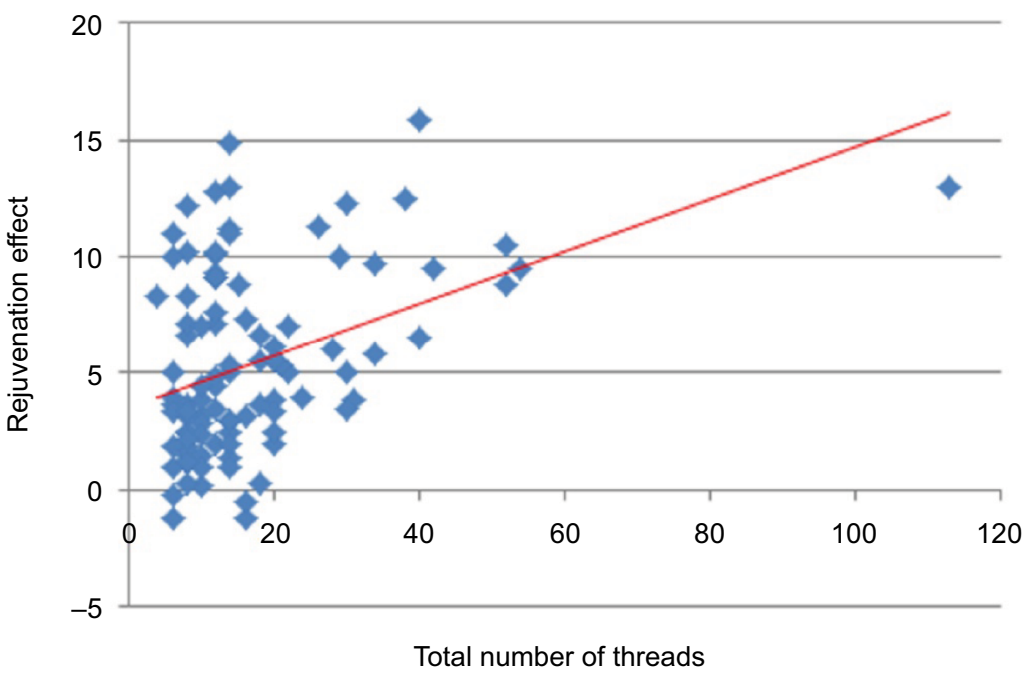

Figure $7 \mathrm{~A}$ scatter plot of the total number of threads used and the rejuvenation effect in 100 patients. Note: The regression line is in red.

the two values ( $P=0.099$, independent $t$-test). Twenty-four out of 100 patients had lipolaser treatment to the neck or perioral area between the first and last visits. The mean rejuvenation effect in these 24 patients was 7.0 \pm 3.1 , whereas in the other 76 , it was $5.0 \pm 4.1$. There was a moderate statistical significance in the difference between the two values $(P=0.015)$.

Forty-eight out of 100 patients underwent Q switch laser treatment for their age spots between the first and last visits. The mean rejuvenation effect of the 48 patients was $6.0 \pm 3.7$, whereas in the other 52 , it was $5.0 \pm 4.2$. There was a weak statistical significance in the difference between the two values $(P=0.103)$.
Large ecchymoses, local infection and protrusion/palpable sensation at the end of the thread were the main complications. The ecchymoses disappeared spontaneously after a few weeks with no treatment required. Local infections occurred at the crossing point of the Xtosis or at the end of the thread around the jaw. In all cases, threads were successfully removed from the infected site by simply pulling them out with forceps, maybe because the tissue had become edematous due to inflammation. The wounds healed, leaving a slight scar. Thread protrusion or palpable thread sensation was treated by partial removal of the thread. A small puncture measuring 1-2 mm was made on the skin and the end of the thread was gently probed and cut off. 
The total number of thread-lifts performed on 100 patients was 289 , and the total number of threads inserted was 1689 . Local infection occurred in three cases. The risk of infection was estimated at $1.0 \%$ of the total number of thread-lifts and $0.17 \%$ of the total number of threads used.

Large ecchymoses occurred in seven cases $(2.4 \%$ of the operations performed and $0.41 \%$ of the threads used) and protrusion or palpable sensation at the end of the thread occurred in 24 cases $(8.3 \%$ of the operations performed and $1.4 \%$ of the threads used). There were no other major complications. No complication associated with or peculiar to the existence of multiple threads present in the tissue for many years was observed.

\section{Discussion}

A unique free software called How Old Net is available online, where one can upload a photo of a face, and a robot, named the How Old Robot, guesses the age. ${ }^{13}$ This software was used on the first visit photos of the 100 study patients, the results of which are plotted in Figure 8. The ages guessed by the robot seemed to be lower than the actual ages. Moreover, the robot could not identify a face without eyes, which is why the author did not use the software in the study. However, in the future, a similar, yet more advanced software may replace the laborious process of patient age guessing adopted in this study.

Another method that can amplify the appearance of sagging is to convert the photo to a monochrome image and increase the contrast. Comparing Figures 1 and 9, one could more easily identify the improvement in sagging in Figure 9 than in Figure 1. However, the disadvantage of this method is that the image in the photo becomes too artificial and the age guessed using the photo might be less realistic.

It is very interesting that the thread-lifting procedure's rejuvenation effect decreased during the first 11 months after the operation and increased 1 year after the procedure. The author considers that there are both early and late effects of the thread-lift procedure. The rejuvenation effect gradually decreased over a period of months over the first year and a late increase becomes more obvious over a period of years. As the insoluble threads function like suspending ligaments in the tissue, the progress of sagging can be prevented after the operation. ${ }^{8}$ As graphically described in Figure 10, the youth value can increase over time.

The tissue is tightened by insoluble threads at the subcutaneous layer. The descended tissue appears prominently lifted, particularly just after the operations, but loosens in months. However, the threads seemed to have a prophylactic effect on sagging from the long-term viewpoint. This is just the conclusion of the paper.

The author admits that there are limitations in the study. As the study design is retrospective, the value of the rejuvenation effect should not be regarded as the mean value of the entire patient population who had undergone the insoluble thread-lift operation. As the enrolled patients were all revisits, they must belong to the group of patients satisfied with the result. Another issue is that the effect of treatments other than the thread-lift was not excluded in the study. However, the influence of hyaluronic acid injection to the nasolabial fold, lipolaser treatment to the neck or perioral region, or

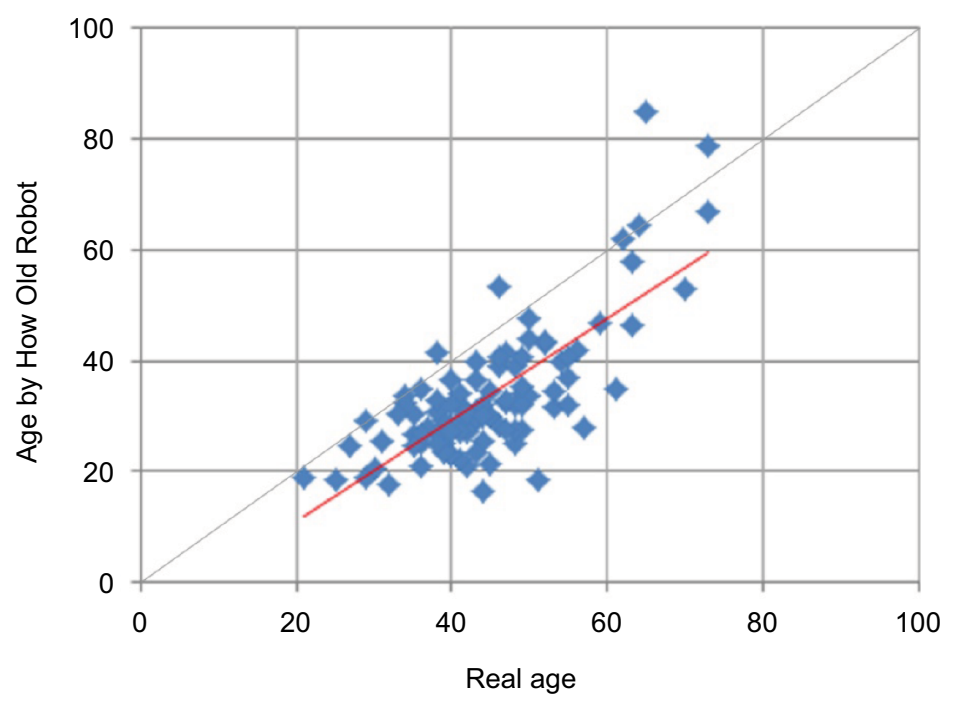

Figure $8 \mathrm{~A}$ scatter plot of the patients' real ages at the first visit and the ages evaluated by How Old Robot ${ }^{13}$ in 100 patients. Note: The regression line is in red. 

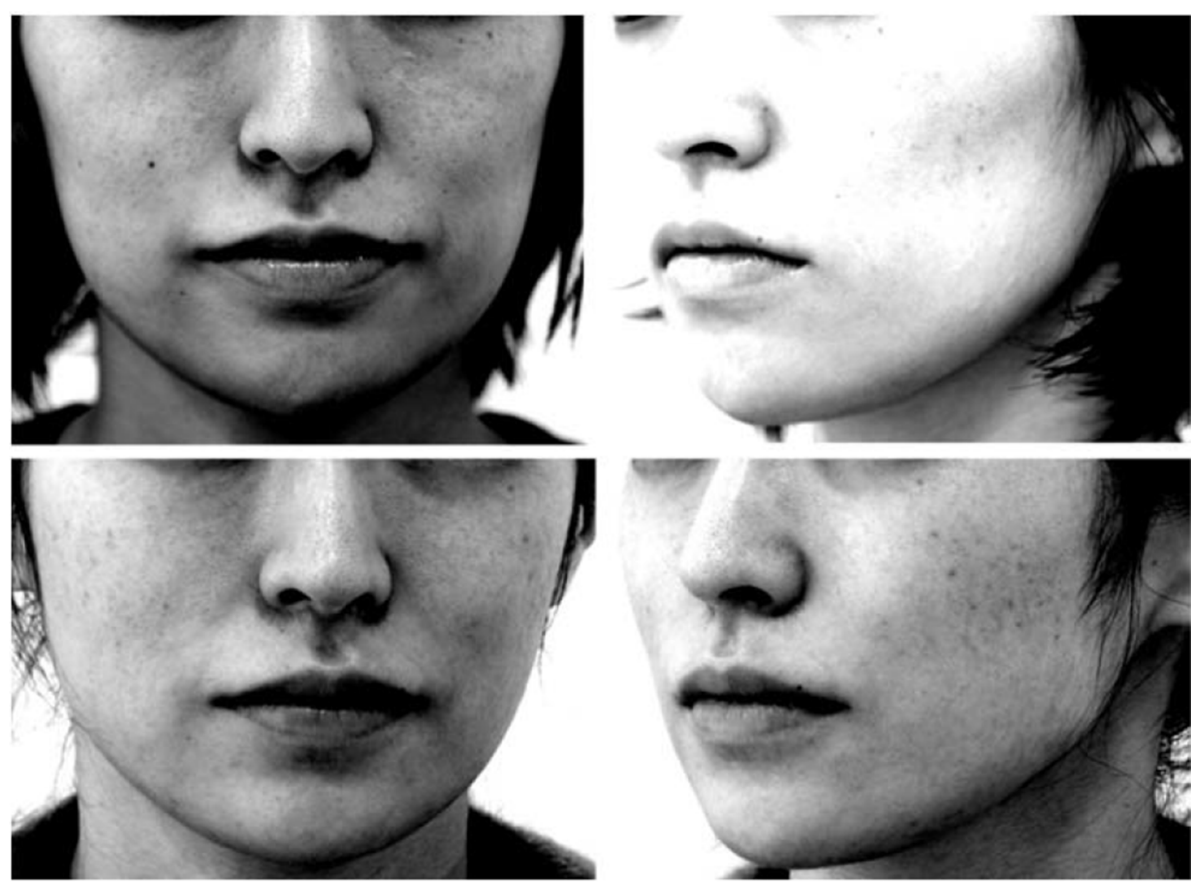

Figure 9 A monochromatic version of the photos in Figure I with intensified contrast.

Notes: The upper photos are from the first visit at the age of 30 , and the lower photos are of the last visit at the age of 34 . The patient underwent six thread-lifting surgeries and the total of 40 threads were inserted. The time from the last operation to the last visit was 9 months. Her apparent age was 31 at the first visit and 29 at the last visit.

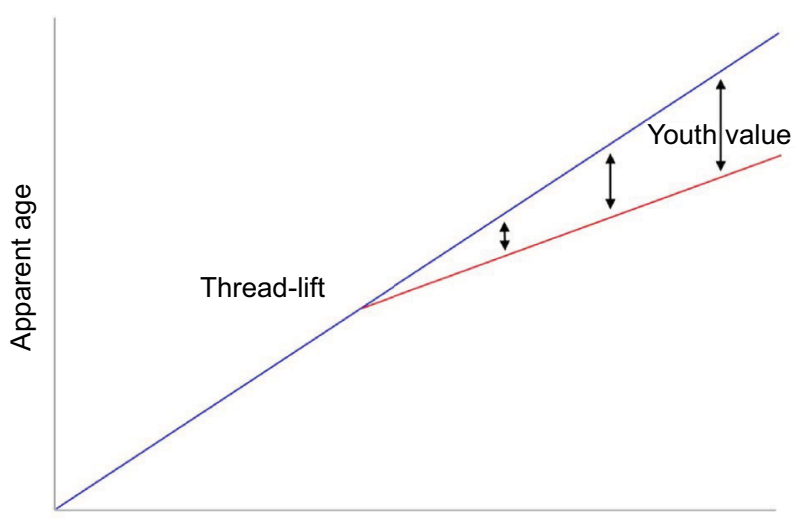

Real age

Figure 10 A diagram showing the hypothesis of why youth value increases years after the thread-lift operation.

Q switch laser treatment for age spots was confirmed to be rather small (an increase of 1.0-2.0 in rejuvenation effect). Of the 100 patients, there were 5 who had a thread-lift operation $>3$ years after the first visit. The photos of the five patients were evaluated at the first visit, just before the first thread-lift operation and at the last visit, and the youth values are plotted in Figure 11. The difference in the rejuvenation effects before and after the thread-lift operation may help to justify the superiority of the thread-lift technique over the other less-invasive treatments. Photos of one of the five cases are presented in Figure 12.

Nevertheless, despite the abovementioned limitations, the author still believes the results of this study can be useful

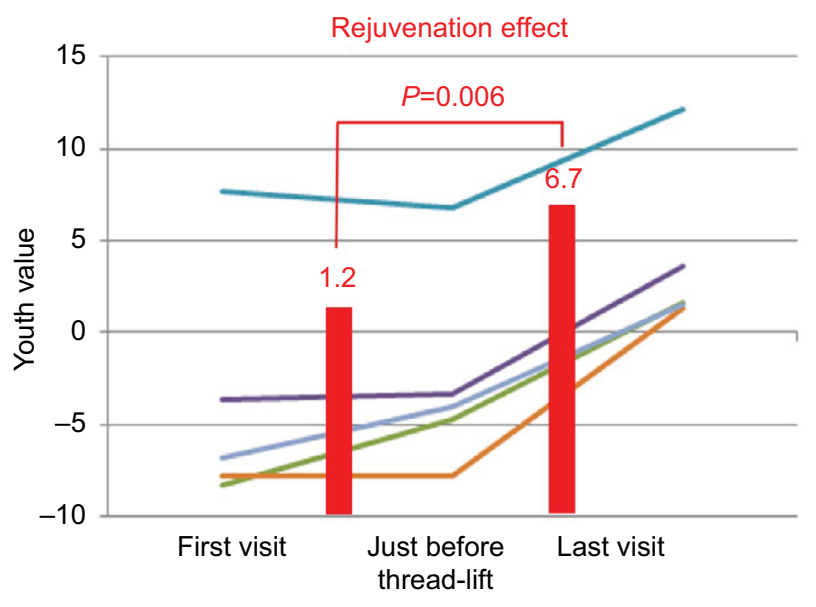

Figure II The change in youth values of the five patients at the first visit, just before the operation of thread-lift and at the last visit.

for practitioners because there is very little information on the long-term prognosis of the thread-lift operation. ${ }^{9-11}$ As this study was conducted in only one clinic and the operation was performed by only one doctor, a multicenter trial would be necessary in the future for generalization of the results in the study.

\section{Conclusion}

The insoluble thread-lifting technique appears to be associated with both early and late effects. The rejuvenation effect appeared to decrease during the first year, but increased thereafter. A multicenter trial is necessary to confirm these findings. 

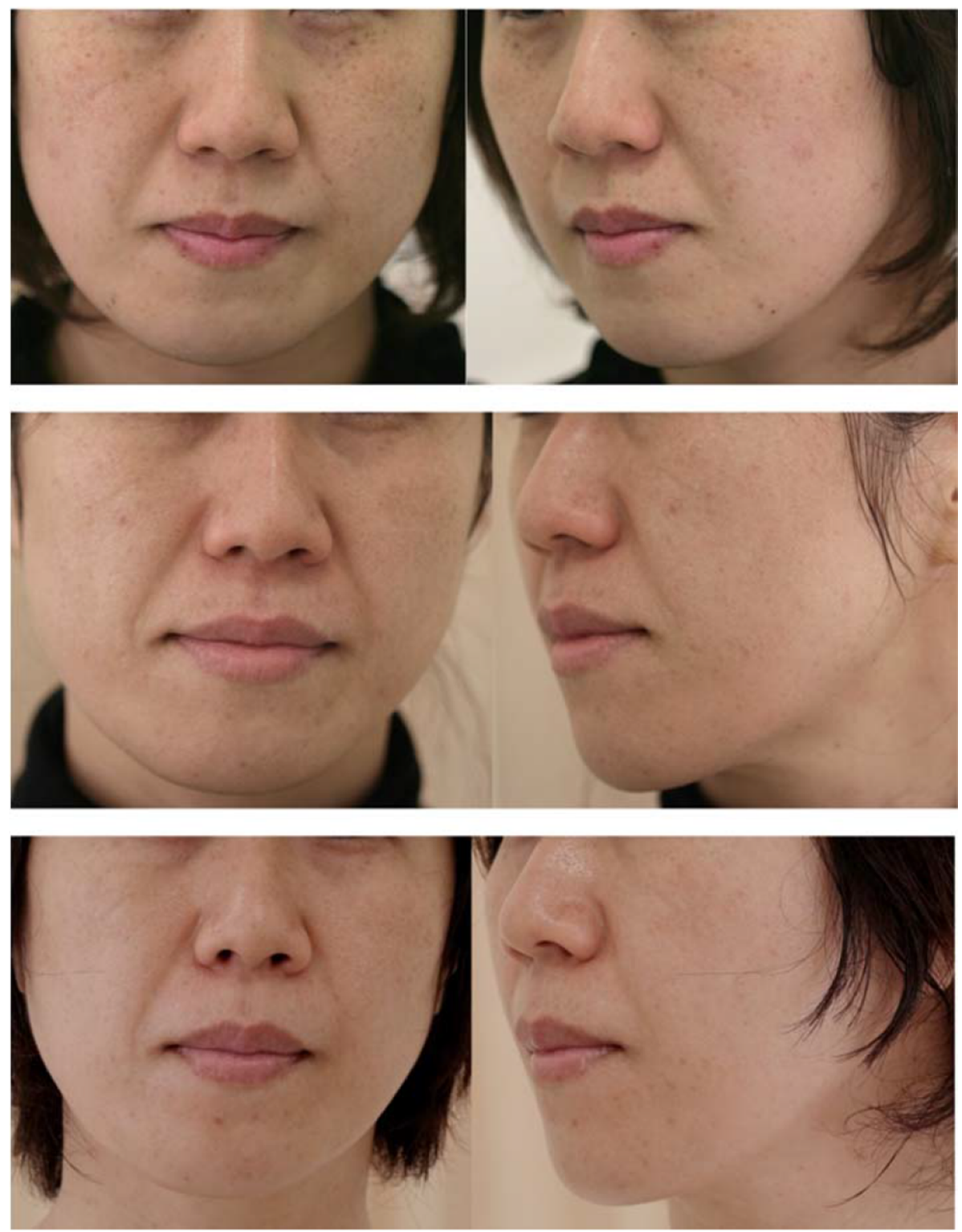

Figure 12 Photos of I of 5 patients who had a thread-lifting operation $>3$ years after the first visit.

Notes: Upper panel: a case at the first visit for age spots removal. The real age was 36 , the apparent age was 42 and the youth value was -6 . Middle panel: the appearance just before the thread-lift operation. The real age was 42 , the apparent age was 46 and the youth value was -4 . Lower panel: three years after the last thread-lift operation. The real age was 44, the apparent age was 42 and the youth value was 2 .

\section{Disclosure}

The author reports no conflicts of interest in this work.

\section{References}

1. Serdev NP. 'Serdev suture' techniques in cosmetic surgery: filos elasticos Serdev. Int J Cosmet Surg. 2006;6(1):408-503.

2. Sasaki GH, Oberg KC, Kim EY. Bidirectional lift of the anterior midcheek with Gore-Tex cable sutures. Aesthet Surg J. 2003;23(4):248-256.

3. Adamyan AA, Sulamanidze MA. Clinical aspects of bloodless facelift using APTOS filaments. Ann Plast Reconstr Aesthet Surg. 2002;2(1):24-34.

4. Sulamanidze M, Sulamanidze G. Facial lifting with Aptos Methods. $J$ Cutan Aesthet Surg. 2008;1(1):7-11.

5. Wu WT. Barbed sutures in facial rejuvenation. Aesthet Surg J. 2004;24(6):582-587.
6. Savoia A, Accardo C, Vannini F, Di Pasquale B, Baldi A. Outcomes in thread lift for facial rejuvenation: a study performed with happy lift ${ }^{\mathrm{TM}}$ revitalizing. Dermatolo Ther (Heidelb). 2014;4(1):103-114.

7. Suh DH, Jang HW, Lee SJ, Lee WS, Ryu HJ. Outcomes of polydioxanone knotless thread lifting for facial rejuvenation. Dermatol Surg. 2015;41(6):720-725.

8. Adamyan AA, Sulamanidze MA, Skuba ND, Khusnutdinova ZR. Morphological foundations of facelift using APTOS filaments. Ann Plast Reconstr Aesthet Surg. 2002;3:19-26.

9. Atiyeh BS, Dibo SA, Costagliola M, Hayek SN. Barbed sutures "lunch time" lifting: evidence-based efficacy. J Cosmet Dermatol. 2010;9(2):132-141.

10. Kaminer MS, Bogart M, Choi C, Wee SA. Long-term efficacy of anchored barbed sutures in the face and neck. Dermatol Surg. 2008;34(8):1041-1047.

11. Garvey PB, Ricciardelli EJ, Gampper T. Outcomes in threadlift for facial rejuvenation. Ann Plast Surg. 2009;62(5):482-485. 
12. YouTube.com [video on the Internet]. Supplemental Digital Content Long term effect of the insoluble thread lifting technique. mototclinic 2017. Available from: https:/www.youtube.com/watch?v=NfIoP3ce0 24\&feature=youtu.be. Accessed November 08, 2017.
13. how-old.net [homepage on the Internet]. How old do I look? Microsoft Azure; 2016. Available from: https://how-old.net/. Accessed November $08,2017$.
Clinical, Cosmetic and Investigational Dermatology is an international, peer-reviewed, open access, online journal that focuses on the latest clinical and experimental research in all aspects of skin disease and cosmetic interventions. This journal is included on PubMed. The manuscript management system is completely online and includes a very quick and fair peer-review system, which is all easy to use. Visit http://www.dovepress.com/testimonials.php to read real quotes from published authors 